\title{
A RESULT ON BEST APPROXIMATION IN LOCALLY CONVEX SPACES
}

\author{
ABDUL LATIF, ARJAMAND BANO AND ABDUL RAHIM KHAN
}

\begin{abstract}
In this paper we obtain a result on best approximation for multivalued nonexpansive maps in locally convex spaces. Our results generalize and extend some known results.
\end{abstract}

\section{Introduction}

Brosowski [1], Meinardus [7], Shahzad [13], Singh [14], [15], used fixed point theory for single-valued maps to prove some interesting results in approximation theory. Fixed point theorem in locally convex topological vector spaces for single and multivalued nonexpansive mappings has been studied in [12], [15], [16], [17], [18], and by many others in the literature. In this paper, we prove two results on best approximation for compact valued nonexpansive maps in the setting of Hausdorff locally convex spaces, which extend some known results.

Throughout this paper, $X$ will denote a complete Hausdorff locally convex topological linear space unless stated otherwise, $Q$ the family of continuous semi-norms generating the topology of $X$ and $K(X)$ the family of nonempty compact subsets of $X$. For each $p \in Q$ and $A, B \in K(X)$, we define

$$
D_{p}(A, B)=\max \left\{\sup _{a \in A} \inf _{b \in B}[p(a-b)], \sup _{b \in B} \inf _{a \in A}[p(a-b)]\right\} .
$$

Although $p$ is only a semi-norm, $D_{p}$ is a Hausdorff metric on $K(X)$.

Note that if $X$ is locally convex space together with a family $Q$ of continuous seminorms and $M$ a subset of $X$, then for each $p \in Q$ and $x \in X, d_{p}(x, M)=\inf _{y \in M} p(x-y)$ and $P_{M}(x)=\left\{z \in M: p(x-z)=d_{p}(x, M)\right\}$ is called the set of best $M$-approximants to $X . P_{M}(x)$ is always bounded subset of $X$ and it is closed or convex if $M$ is closed or convex (see [1]).

A multivalued map $T: M \rightarrow 2^{X}$ (the collection of nonempty subsets of $X$ ) is called demiclosed if for every sequence $\left\{x_{n}\right\} \subset M$ and any $y_{n} \in T\left(x_{n}\right), n=1,2,3, \ldots$, such that $x_{n} \stackrel{w}{\longrightarrow} x$ and $y_{n} \rightarrow y$, we have $x \in M$ and $y \in T(x)$.

Received February 24, 2004; revised October 7, 2004.

2000 Mathematics Subject Classification. 41A50, 47H10, 54H25.

Key words and phrases. Locally convex spaces, fixed points, best $M$-approximations, invariant approximation, multivalued nonexpansive map, demiclosed operator. 
Let $M$ be a nonempty sbuset of $X$. A mapping $T: M \rightarrow K(M)$ is called multivalued contraction if for a fixed constant $k_{p}, 0<k_{p}<1$ such that for each $x, y \in M$ and for each $p \in Q$, we have

$$
D_{p}(T(x), T(y)) \leq k_{p} p(x-y) .
$$

The map $T$ is called nonexpansive if for each $x, y \in M$ and $p \in Q$,

$$
D_{p}(T(x), T(y)) \leq p(x-y) .
$$

An element $x \in X$ is called a fixed point of multivalued map $T$ if

$$
x \in T(x) .
$$

We denote by $F(T)$ the set of fixed points of $T . M$ is said to be starshaped with respect to a point $q \in M$ if for each $x \in M$, the segment joining $x$ to $q$ is contained in $M$ (that is, $\lambda x+(1-\lambda) q \in M$ for each $x \in M$, and real $\lambda$ with $0 \leq \lambda \leq 1)$.

$M \subset X$ is said to be starshaped if it is starshaped with respect to one of its element. A covnex set is obviously starshaped, but starshaped need not be convex.

A space $X$ is said to satisfy Opial's property [8] if for every sequence $\left\{x_{n}\right\}$ in $X$ weakly convergent to $x \in X$, then for each $p \in Q$, the inequality

$$
\liminf p\left(x_{n}-x\right)<\liminf p\left(x_{n}-y\right)
$$

holds for all $y \neq x$.

It is well known that all Hilbert spaces and $l_{p}(1<p<\infty)$ spaces satisfy Opial's property while $L_{p}[0,2 \pi](p \neq 2)$ do not (see [2], [4], [8]).

Let $T: M \subseteq X \rightarrow K(X)$. A best approximation $z$ in $M$ to an element $x_{0}$ in $X$ such that $T\left(x_{0}\right)=\left\{x_{0}\right\}$ is called an invariant approximation in $X$ to $x_{0}$ if $z \in T(z)$.

In 1980 Singh [15] proved the following result on best approximation for single-valued nonexpansive map in a locally convex space.

Theorem 1.1. Let $X$ be locally convex linear Hausdorff space and let $f: X \rightarrow X$ be a continuous mapping. Let $M$ be $f$-invariant set and $x_{0}$ an $f$-invariant point. If for every $p$ in $Q$, the set $P_{M}\left(x_{0}\right)$ of best $M$-approximation to $x_{0}$ with respect to $p$ is nonempty, compact and starshaped, $f$ satisfies

$$
p\left(f(x)-f\left(x_{0}\right)\right) \leq p\left(x-x_{0}\right) \quad \text { for all } x \text { in } P_{M}\left(x_{0}\right),
$$

and $f$ is p-nonexpansive on $P_{M}\left(x_{0}\right)$, then $f$ has a fixed point which is a best $M$-approximation to $x_{0}$ in $M$.

In [4], Lami Dozo proved the following useful result, which is a special case of Lemma 3.1 in $[6]$.

Lemma 1.2. Let $M$ be a nonempty weakly compact subset of a Banach space $X$ satisfying Opial's property. Let $T: M \rightarrow K(X)$ be nonexpansive multivalued map. Then $I-T$ is demiclosed. 
We shall need the following result, which contains Lemma 1.2 and is a special case of Lemma 2.5 [3]. For the sake of completeness we give the proof.

Lemma 1.3. Let $M$ be a nonempty weakly sequentially compact subset of a Hausdorff locally convex space $X$ satisfying Opial's property. Let $T: M \rightarrow K(X)$ be nonexpansive multivalued map. Then $I-T$ is demiclosed, where $I$ is the identify map on $M$.

Proof. Let $\left\{x_{n}\right\}$ be a sequence in $M$ and $y_{n} \in(I-T) x_{n}$ be such that $x_{n} \stackrel{w}{\longrightarrow} x$ and $y_{n} \rightarrow y$. Obviously $x \in M$. As $y_{n} \in x_{n}-T\left(x_{n}\right)$, therefore we have

$$
y_{n}=x_{n}-u_{n} \quad \text { for some } u_{n} \in T\left(x_{n}\right) .
$$

Since $T(x)$ is compact set, there is a $v_{n} \in T(x)$ such that for all $p \in Q$,

$$
p\left(u_{n}-v_{n}\right) \leq D_{p}\left(T\left(x_{n}\right), T(x)\right),
$$

But by the nonexpansiveness of $T$, we have

$$
D_{p}\left(T\left(x_{n}\right), T(x)\right) \leq p\left(x_{n}-x\right)
$$

Thus

$$
p\left(u_{n}-v_{n}\right) \leq p\left(x_{n}-x\right) \text { for all } p \in Q .
$$

From (1.1) and (1.2), passing to the limit with resepect to $n$, we obtain

$$
\begin{aligned}
\liminf p\left(x_{n}-x\right) & \geq \liminf p\left(u_{n}-v_{n}\right) \\
& =\liminf p\left(x_{n}-y_{n}-v_{n}\right), \quad \text { for all } p \in Q .
\end{aligned}
$$

By the compactness of $T(x)$, for a convenient subsequence still denoted by $\left\{v_{n}\right\}$, we have $v_{n} \rightarrow v \in T(x)$. Then (1.3) yields

$$
\liminf p\left(x_{n}-x\right) \geq \liminf p\left(x_{n}-y-v\right) \text { for all } p \in Q .
$$

Since $X$ satisfies Opial's property and $x_{n} \stackrel{w}{\longrightarrow} x$, so $x=y+v$. Thus $y=x-v \in x-T(x)=$ $(I-T) x$, which proves that $I-T$ is demiclosed.

\section{Main Results}

Here, we extend Theorem 1.1 to multivalued nonexpansive maps in the setting of a locally convex Hausdorff space.

Theorem 2.1. Let $X$ be a complete locally convex Hausdorff space and $T: X \rightarrow$ $K(X)$ be a multivalued nonexpansive map such that $T\left(x_{0}\right)=\left\{x_{0}\right\}$ for some $x_{0} \in X$. Let $M$ be a nonempty $T$-invariant subset of $X$. For every $p \in Q$, assume that $P_{M}\left(x_{0}\right)$ is nonempty weakly sequentially compact and starshaped. If $I-T$ is demiclosed on $P_{M}\left(x_{0}\right)$, then $P_{M}\left(x_{0}\right) \cap F(T) \neq \emptyset$. 
Proof. Let $D=P_{M}\left(x_{0}\right)$ and let $u \in D$. Then $u \in M$ and

$$
p\left(x_{0}-u\right)=d_{p}\left(x_{0}, M\right) .
$$

Let $v \in T(u) \subset M$. Then we have that

$$
p\left(v-x_{0}\right) \leq D_{p}\left(T(u), T\left(x_{0}\right)\right),
$$

so, by the nonexpansiveness of $T$, we get

$$
D_{p}\left(T(u), T\left(x_{0}\right)\right) \leq p\left(u-x_{0}\right)
$$

Thus

$$
p\left(v-x_{0}\right) \leq p\left(u-x_{0}\right)=d_{p}\left(x_{0}, M\right),
$$

which gives that $v \in D$ and thus $T(u) \subset D$. Therefore $T$ carries $D$ into $K(D)$. Now let $q$ be starcenter of $D$. Then for each $x \in D$ and any $\lambda(0<\lambda<1),(1-\lambda) q+\lambda x \in D$.

Take a sequence $\left\{\lambda_{n}\right\}$ of real numbers such that $0<\lambda_{n}<1$ and $\lambda_{n} \rightarrow 1$ as $n \rightarrow \infty$. Now for each $n$ define a multivalued map $T_{n}$ by

$$
T_{n}(x)=\left(1-\lambda_{n}\right) q+\lambda_{n} T(x), \quad \text { for all } x \in D .
$$

Clearly, each $T_{n}$ maps $D$ into $K(D)$. Furthermore, for any $x, y \in D$ we have

$$
D_{p}\left(T_{n}(x), T_{n}(y)\right)=\lambda_{n} D_{p}(T(x), T(y)),
$$

so, by the nonexpansiveness of $T$, we have

$$
D_{p}(T(x), T(y)) \leq p(x-y)
$$

Thus

$$
D_{p}\left(T_{n}(x), T_{n}(y)\right) \leq \lambda_{n} p(x-y),
$$

which proves that each $T_{n}$ is a contraction for every $p$ in $Q$. By Theorem 3.1 [9], there exists $x_{n} \in D$ such that $x_{n} \in T_{n}\left(x_{n}\right)=\left(1-\lambda_{n}\right) q+\lambda_{n} T\left(x_{n}\right)$ this implies that there is a $y_{n} \in T\left(x_{n}\right)$ such that

$$
x_{n}=\left(1-\lambda_{n}\right) q+\lambda_{n} y_{n} .
$$

As $D$ is weakly sequentially compact, so for a convenient subsequence still denoted by $\left\{x_{n}\right\}$, we have $x_{n} \stackrel{w}{\longrightarrow} z \in D$. Now

$$
x_{n}-y_{n}=\left(1-\lambda_{n}\right)\left(q-y_{n}\right) .
$$

Put $z_{n}=x_{n}-y_{n} \in(I-T) x_{n}$. Then $z_{n} \rightarrow 0$ as $n \rightarrow \infty$. Since $I-T$ is demiclosed on $D$, it follows that $0 \in(I-T) z$, that is, $z \in F(T)$.

Remark 2.2. Theorem 2.1 generalizes Theorem 3 [5] from the Banach space setting to the case of a complete locally convex Hausdorff space. 
Now by Lemma 1.3, we have the following result on invariant approximation for a locally convex Hausdorff space, which satisfies Opial's property.

Corollary 2.3. Let $X$ be a complete locally convex Hausdorff space satisfying Opial's property, and $T: X \rightarrow K(X)$ be a multivalued nonexpansive map such that $T\left(x_{0}\right)=$ $\left\{x_{0}\right\}$ for some $x_{0} \in X$. Let $M$ be a nonempty $T$-invariant subset of $X$. If for every $p \in Q$, the set $P_{M}\left(x_{0}\right)$ is nonempty weakly sequentially compact and starshaped, then $P_{M}\left(x_{0}\right) \cap F(T) \neq \phi$.

Proof. Let $D=P_{M}\left(x_{0}\right)$ and let $u \in D$. Then $u \in M$ and

$$
p\left(x_{0}-u\right)=d_{p}\left(x_{0}, M\right) .
$$

Let $v \in T(u) \subset M$. Then we have that

$$
p\left(v-x_{0}\right) \leq D_{p}\left(T(u), T\left(x_{0}\right)\right),
$$

so, by the nonexpansiveness of $T$, we get

$$
D_{p}\left(T(u), T\left(x_{0}\right)\right) \leq p\left(u-x_{0}\right) .
$$

Thus

$$
p\left(v-x_{0}\right) \leq p\left(u-x_{0}\right)=d_{p}\left(x_{0}, M\right),
$$

which gives that $v \in D$ and thus $T(u) \subset D$. Therefore $T$ carries $D$ into $K(D)$. Thus by Lemma 1.3, $I-T$ is demiclosed on $D$. Now the result follows from Theorem 2.1.

\section{References}

[1] B. Brosowski, Fixpunktsatze in der approximation theorie, Mathematica (Cluj) 11(1969), 195-220.

[2] J. P. Gosses and E. Lami Dozo, Some geometric properties related to the fixed point theory for nonexpansive mappings, Pacific J. Math. 40(1972), 565-573.

[3] A. R. Khan, A. Latif, N. Nawab and A. Bano, Coincidence point results in locally convex spaces, Internat. J. Pure Appl. Math. 3 (2002), 413-423.

[4] E. Lami Dozo, Multivalued nonexpansive mappings and Opial's condition, Proc. Amer. Math. Soc. 38(1973), 286-292.

[5] A. Latif and A. Bano, A result on invariant approximation, Tamkang J. Math. 33(2002), 89-92.

[6] A. Latif and I. Tweddle, Some results on coincidence points, Bull. Austral. Math. Soc. 59(1999), 111-117.

[7] G. Meinardus, Invarianze bei linearen approximation, Arch. Rational Mech. Anal. 14 (1963), 301-303.

[8] Z. Opial, Weak convergence of successive approximations for nonexpansive mappings, Bull. Amer. Math. Soc. 73(1967), 531-537.

[9] D. V. Pai and P. Veeramani, Some fixed point theorems for multi-mappings, Indian j. Pure Appl. Math. 14 (1983), 1157-1165. 
[10] S. Reich, Approximation selection best approximation fixed points and invariant set, J. Math. Anal. 62(1978), 104-113.

[11] S. A. Sahab, M. S. Khan and S. Sessa, A result in best approximation theory, J. Approx. Theory 55(1988), 349-351.

[12] B. N. Sahney, K. L. Singh and J. H. M. Whitfield, Best approximation in locally convex spaces, J. Approx. Theory. 38(1983), 182-187.

[13] N. Shahzad, A result on best approximation, Tamkang J. Math. 29(1998), 223-226.

[14] S. P. Singh, Application of fixed point theorem to approximation theory, J. Approx. Theory 25(1979), 88-89.

[15] S. P. Singh, Some results on best approximation in locally convex spaces, J. Approx. Theory. 28(1980), 329-332.

[16] K. L. Singh and Y. Chen, Fixed points for nonexpansive multivalued mapping in locally convex spaces, Math. Japon 36(1991), 423-425.

[17] K. L. Singh and J. H. M. Whitefield, Fixed points for single valued and multivalued functions, operator eqations and fixed point theroems, The MSRI-Koree Publication 13 (1986), 121126.

[18] C. H. Su and V. M. Sehgal, Some fixed point theorems for nonexpansive multivalued functions in locally convex spaces, Academia Sinica 4(1976), 49-52.

Department of Mathematics, King Abdul Aziz University, P.O.Box 80203, Jeddah-21589. Saudi Arabia.

E-mail: abdul_latifn@hotmail.com

Department of Mathematics, Gomal University, Dera Ismail Khan, Pakistan.

E-mail: arjamandbano2002@yahoo.com

Department of Mathematical Sciences, King Fahad University of Petroleum and Minerals, Dhahran-31261-Saudi Arabia.

E-mail: arahim@kfupm.edu.com 